\title{
Dissemination and visualisation of reference decay data from Decay Data Evaluation Project (DDEP)
}

\author{
Christophe Dulieu ${ }^{\mathrm{a}}$, Mark A. Kellett, and Xavier Mougeot \\ CEA, LIST, Laboratoire National Henri Becquerel (LNE-LNHB), Bât. 602, PC 111, CEA-Saclay, 91191 Gif-sur-Yvette Cedex, France
}

\begin{abstract}
As a primary laboratory in the field of ionising radiation metrology, the Laboratoire National Henri Becquerel (LNE-LNHB), CEA Saclay, is involved in measurements, evaluations and dissemination of radioactive decay data.

Data measurements undertaken by various laboratories are evaluated by an international commission of experts (Decay Data Evaluation Project) coordinated by LNHB staff in order to establish a set of recommended decay scheme data.

New nuclide evaluations are regularly added to our website, the NUCLÉIDE database, published in the BIPM-5 Monographie series and uploaded to our web application LARAWEB, a dedicated tool for alpha and gamma spectrometry. The Mini Table of Radionuclides is produced from time-to-time with data extracted from our database.

Various publications are described, along with new search criteria and decay scheme visualisation in LARAWEB.
\end{abstract}

\section{Introduction}

Evaluation of nuclear decay data at the Laboratoire National Henri Becquerel (LNHB), CEA, began in 1974 and many publications on this topic have been released. In order to facilitate the management and distribution of these data, a computerized database called NUCLÉIDE was developed, as well as many tools for both evaluators and users.

All necessary data are stored in one single database, NUCLÉIDE, which is used as an entry point and has allowed the development of specific software to maintain and update this database. The LNHB is therefore able to offer a variety of tools related to reference nuclear decay data for users in many fields such as nuclear medicine, reactor monitoring and shielding, spectrometry, environmental studies, etc. We present some of the publications and tools we distribute, and more specifically the latest enhancements of LARAWEB which is our web application for alpha and gamma spectrometry.

\section{Data evaluation}

New or updated nuclide evaluations are regularly added to the NUCLÉIDE database through the work of evaluators from the Decay Data Evaluation Project (DDEP) [1]. These evaluations are undertaken when new measurements are published or special interest for a new nuclide emerges. All published measurements and data are analysed and compiled by a DDEP evaluator and a set of recommended decay data is established. After a review procedure among evaluators, these data are inserted in the NUCLÉIDE

$\bar{a}$ e-mail: christophe.dulieu@cea.fr database and made available to the users through multiple media: electronic files on our website, printed publications, and our web applications.

\section{LNHB website}

In the atomic and nuclear data section of our website, one can find a variety of information concerning evaluated nuclides, mainly in the form of PDF or text files. Thus, the recommended data and decay scheme relative to a nuclide, and information concerning the way the evaluation was achieved, are available as PDF files. Decay data are also available in the ENSDF file format [2] and as a simple text file. A specific PenNuc text file, intended to be used for Penelope Monte Carlo simulation, has recently been added that describes the complete decay scheme of a nuclide to be used as a radioactive source in a simulation process.

As of today, nearly 220 nuclides are available, and the website is regularly updated when new evaluations become available. The main LNHB website can be found at: http://www.nucleide.org/ with the Atomic and Nuclear Data Section at: http://www.nucleide.org/ NucData.htm.

\section{Monographie BIPM-5 series}

All the latest evaluated nuclides are periodically published in a printed monograph under the auspices of the BIPM (Bureau International des Poids et Mesures) [3], as well as electronically in a PDF file format available on the BIPM website [4]. The eighth volume in the series has been published by the end of 2016 and contains 32 nuclide evaluations. Comments on how these evaluations were conducted is available online as PDF files.

(c) The Authors, published by EDP Sciences. This is an Open Access article distributed under the terms of the Creative Commons Attribution License 4.0 (http://creativecommons.org/licenses/by/4.0/). 


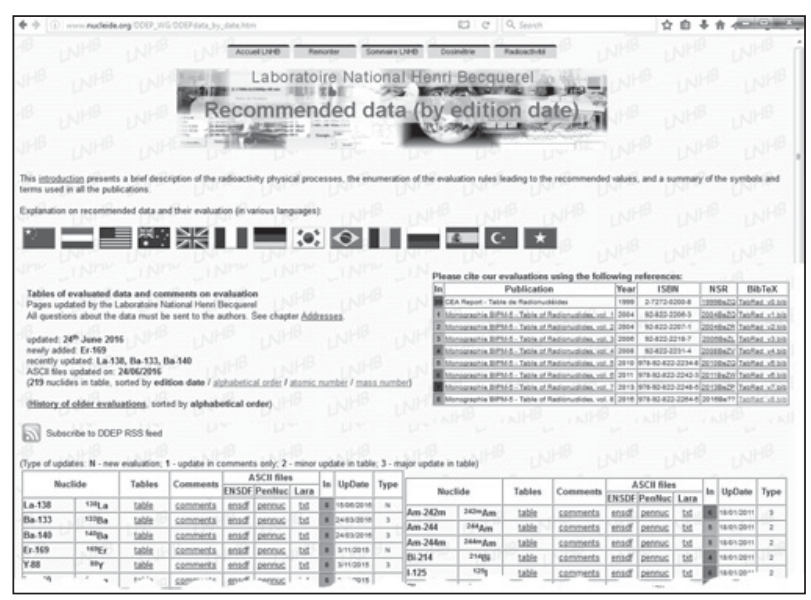

Figure 1. Extract of recommended decay data from the LNHB website.

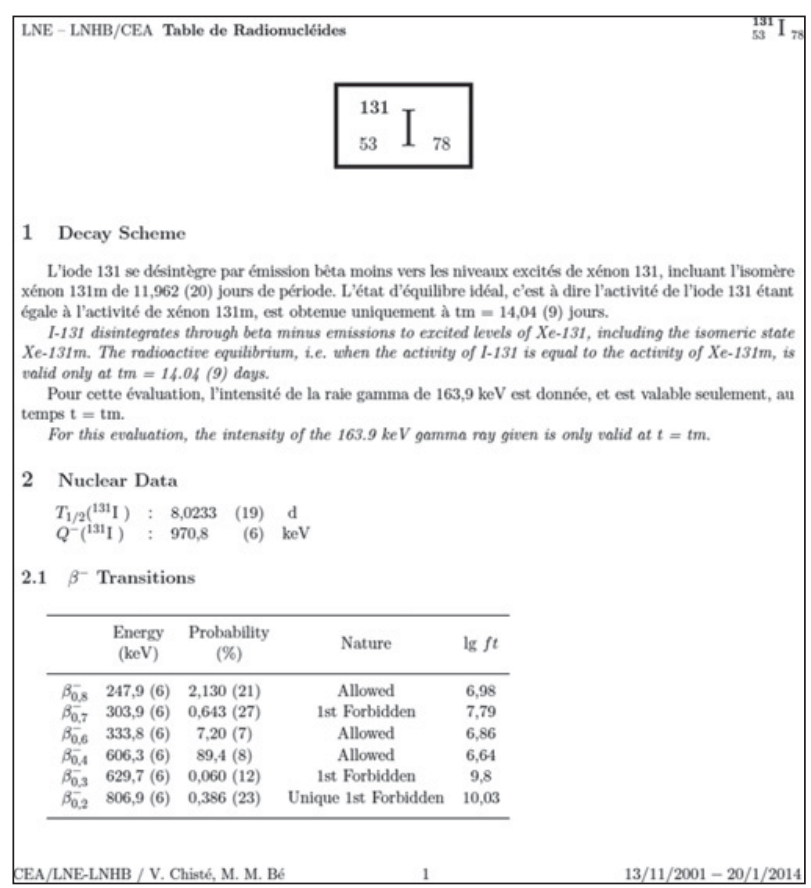

Figure 2. Extract of nuclide data from Monographie BIPM-5.

\section{Mini Table of Radionuclides}

At the end of 2015, the LNHB published a new and updated edition of its booklet version of the Table of Radionuclides known as the "Mini Table of Radionuclides". This work presents, in a concise way, the parameters characterizing each radionuclide; the half-life and the main alpha, beta, gamma and X-ray emissions, and daughters for a selection of about 300 nuclides of particular interest in the fields of spectrometry, medicine and practical industrial applications [5].

\section{LARAWEB/NUCLÉIDE - LARA}

LARAWEB is a web-based application designed for spectrometry purposes. It contains information relating to $\approx 450$ nuclides of particular interest in the field of alpha, $\mathrm{X}$ - and gamma-ray spectrometry. The main data presented

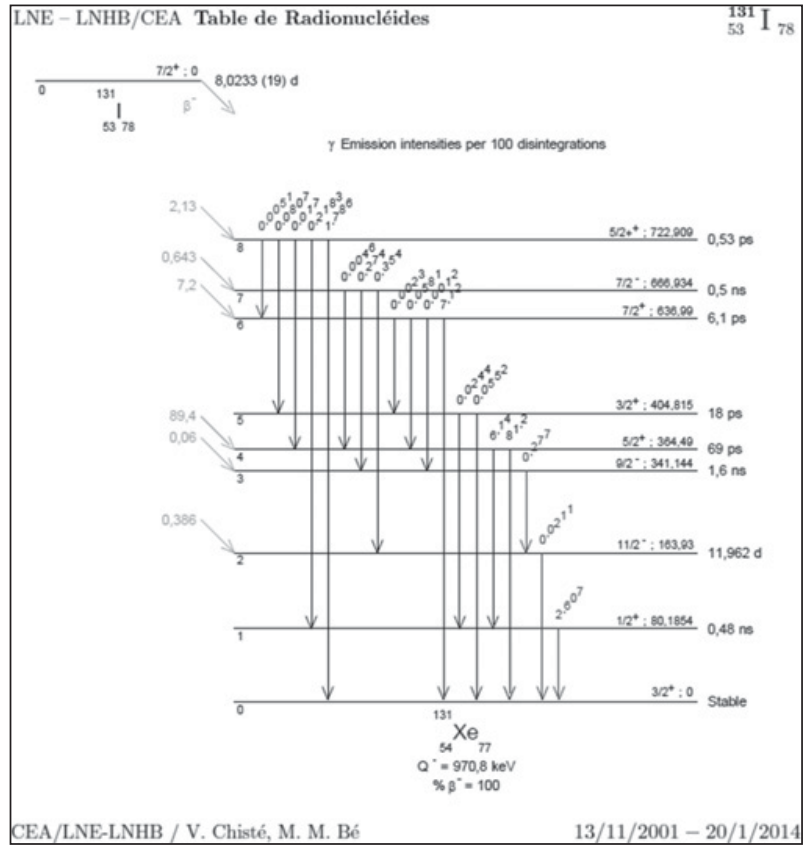

Figure 3. Nuclide decay scheme extracted from Monographie BIPM-5.

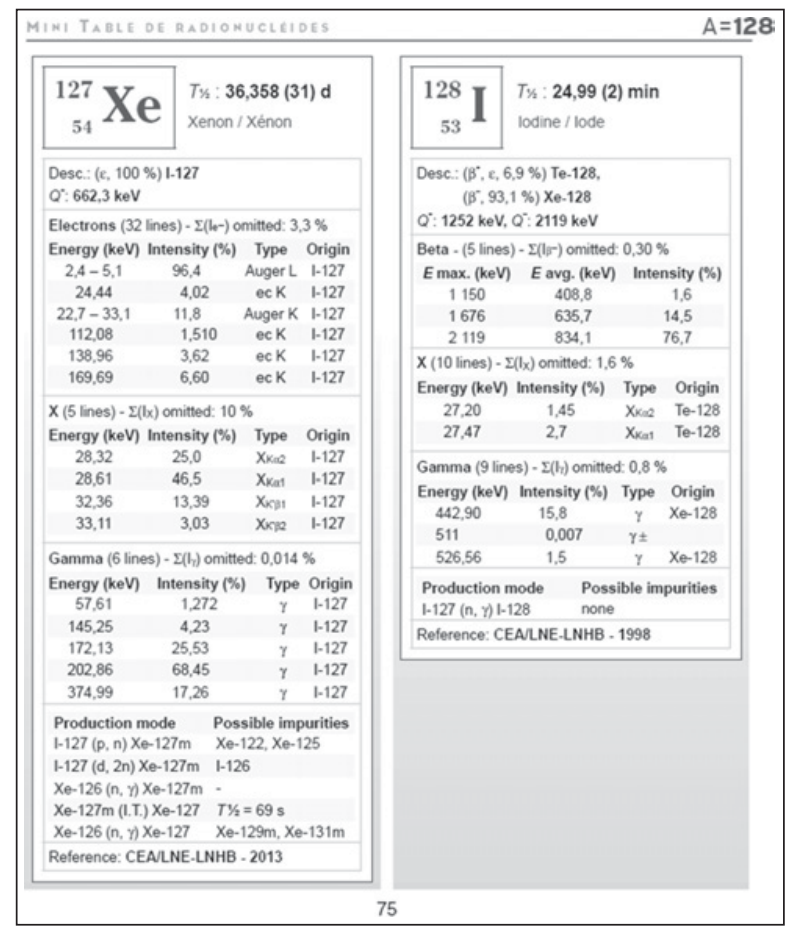

Figure 4. Extract from the Mini Table (2015 version).

are half-life, decay constant, daughters, $\mathrm{Q}$ values, possible parents, specific activity and, most importantly, alpha, X and gamma emissions for each selected nuclide.

\subsection{Decay data display}

Energy and intensity thresholds can be set to restrict the displayed data to the most intense emissions. Multiple nuclides can be selected at the same time for comparison. When available, starting and ending levels of each emission are also displayed. Such information now allows the user to interactively display the nuclide decay scheme, 


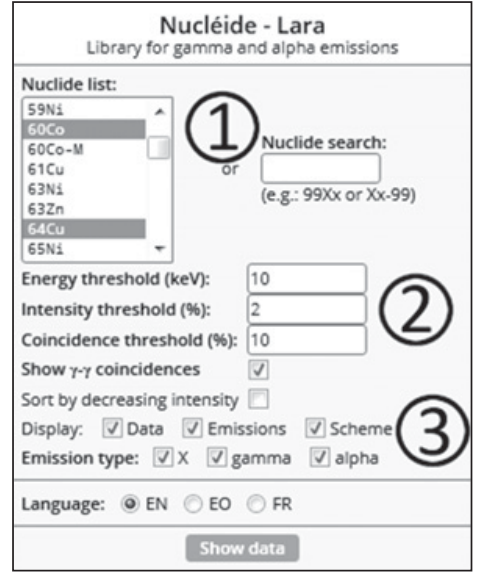

Figure 5a. Main LARAWEB window (1). selection of nuclides to be displayed. (2): optional thresholds settings. (3): selection of information to be displayed.

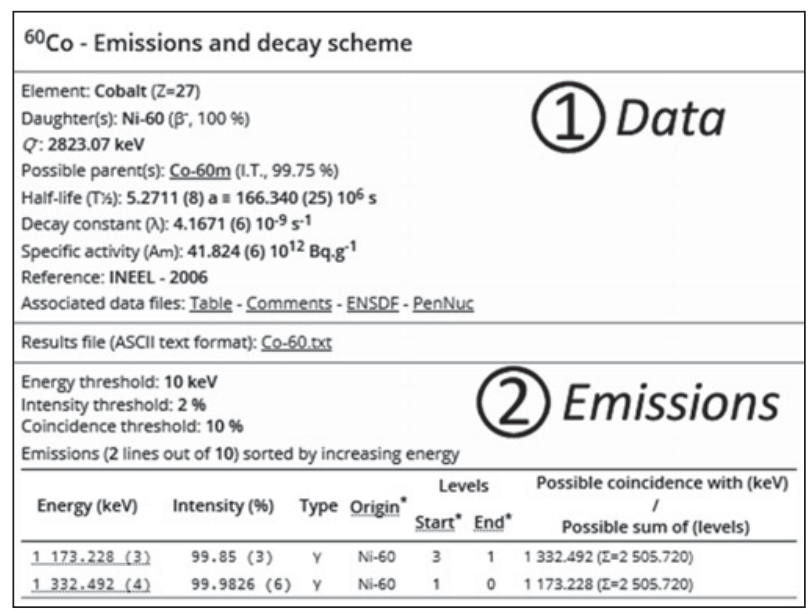

Figure 5b. LARAWEB data window. (1): Main data relating to the selected nuclide(s). (2): associated emissions according to thresholds.

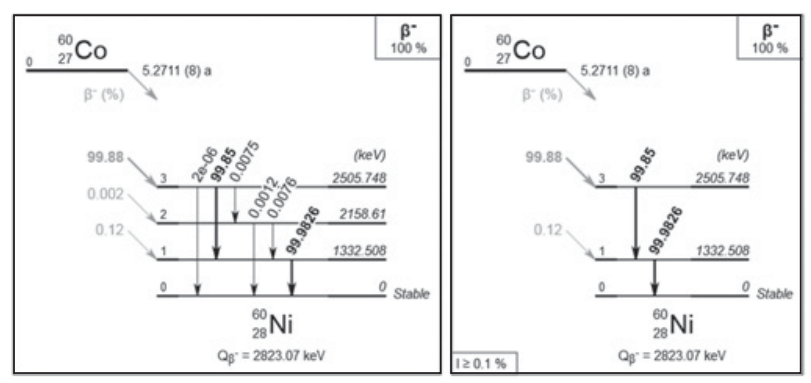

Figure 5c. LARAWEB decay scheme plot. Left: complete scheme. Right: reduced scheme based on selected thresholds.

according to the selected thresholds. Thus, only the most intense or energetic emissions are displayed, offering a simplified view of the decay scheme.

LARAWEB can display the possible coincidences between to cascading nuclides, based on their respective intensities. This feature, combined with the possibility to plot a simplified decay scheme, makes it a convenient tool for spectrometrists.

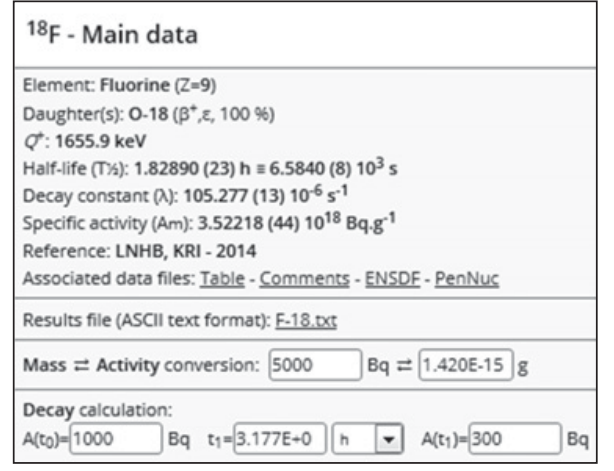

Figure 6. Mass to activity conversion, and simple decay calculation tools in LARAWEB.

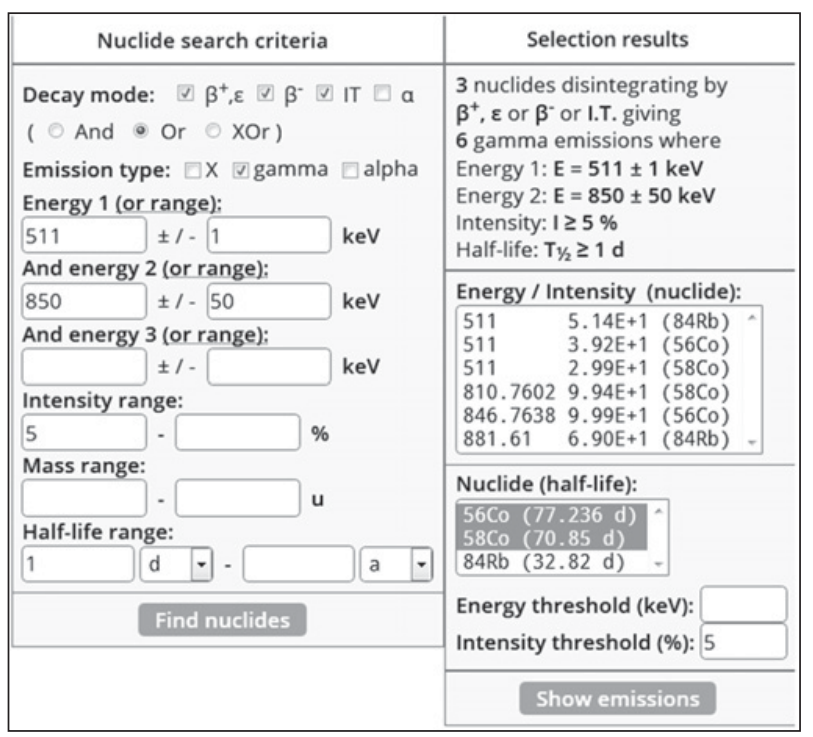

Figure 7a. LARAWEB search engine: Left: search criteria Right: corresponding nuclides, ordered by energy or half-life.

\subsection{Conversion and decay tools}

New calculation tools have also been added to the application. A mass to activity converter calculates the activity of a sample for a given mass and nuclide, considered as pure, and vice versa. A decay tool gives the activity of a nuclide after a time $t_{1}$ knowing the activity at $t_{0}$ or reciprocally, the time $t_{1}$ needed to obtain a given activity.

\subsection{Advanced search engine}

An integrated search engine allows all nuclides to be found which correspond to a range of chosen search criteria, such as; decay mode $\left(\beta^{+} / \varepsilon, \beta^{-}\right.$, IT, $\alpha$ ), emission type (alpha, $\mathrm{X}$ or gamma), emission energies (up to three individual energies or ranges may be specified), emission intensity, mass number and half-life. The user can then select one or more nuclides amongst the resulting list in order to display the complete information. Emissions meeting the search criteria are then highlighted, along with other significantly intense emissions.

This tool can be used to quickly identify the originating radionuclide of an alpha or gamma ray in a spectrum when the energies of the peaks are known.

LARAWEB is available through the Atomic and Nuclear Data section of the LNHB website: http: //www . nucleide.org/NucData.htm or directly at the following address: http://www. nucleide.org/Laraweb. 


\begin{tabular}{|c|c|c|c|c|c|}
\hline \multicolumn{6}{|c|}{${ }^{56}$ Co - Emissions } \\
\hline \multicolumn{6}{|c|}{ Element: Cobalt $(\mathrm{Z}=27)$} \\
\hline \multicolumn{6}{|c|}{ Daughter(s): Fe-56 ( $\left.\beta^{+}, \varepsilon, 100 \%\right)$} \\
\hline \multicolumn{6}{|l|}{$Q^{+}: 4566 \mathrm{keV}$} \\
\hline \multicolumn{6}{|c|}{ Possible parent(s): Ni-56 $\left(\beta^{+}, \varepsilon, 100 \%\right)$} \\
\hline \multicolumn{6}{|c|}{ Half-life (T/2): $77.236(26) \mathrm{d} \equiv 6.6732(22) 10^{6} \mathrm{~s}$} \\
\hline \multicolumn{6}{|c|}{ Decay constant $(\lambda): 103.870(35) 10^{-9} \mathrm{~s}^{-1}$} \\
\hline \multicolumn{6}{|c|}{ Specific activity (Am): 1.11700 (38) $10^{15}$ Bq. $\mathrm{g}^{-1}$} \\
\hline \multicolumn{6}{|c|}{ Reference: LBNL, NPL - 2005} \\
\hline \multicolumn{6}{|c|}{ Associated data files: Table - Comments - ENSDF- PenNuc } \\
\hline \multirow{2}{*}{\multicolumn{6}{|c|}{$\begin{array}{l}\text { Intensity threshold: } 5 \% \\
\text { Gamma emissions ( } 8 \text { lines out of } 47 \text { ) sorted by increasing energy }\end{array}$}} \\
\hline & es out of 47 ) sor & ed by in & ncreasing & energy & \\
\hline \multicolumn{6}{|c|}{ Emissions meeting search criteria Other significantly intense emissions } \\
\hline \multirow{2}{*}{ Energy (keV) } & \multirow{2}{*}{ Intensity (\%) } & \multirow{2}{*}{ Type } & \multirow{2}{*}{ Origin* } & \multicolumn{2}{|c|}{ Levels } \\
\hline & & & & Start ${ }^{*}$ & End ${ }^{*}$ \\
\hline $511(-)$ & $39.21(22)$ & $y \pm$ & Fe-56 & -1 & -1 \\
\hline $846.7638(19)$ & $99.9399(23)$ & y & Fe-56 & 1 & 0 \\
\hline $1037.8333(24)$ & $14.03(5)$ & y & $\mathrm{Fe}-56$ & 5 & 2 \\
\hline $1238.2736(22)$ & $66.41(16)$ & y & $\mathrm{Fe}-56$ & 2 & 1 \\
\hline $1771.327(3)$ & $15.45(4)$ & y & Fe-56 & 8 & 2 \\
\hline $2034.752(5)$ & $7.741(13)$ & y & Fe-56 & 11 & 2 \\
\hline $2598.438(4)$ & $16.96(4)$ & y & Fe-56 & 7 & 1 \\
\hline $3253.402(5)$ & $7.87(3)$ & y & Fe-56 & 10 & 1 \\
\hline \multicolumn{6}{|c|}{${ }^{58}$ Co - Emissions } \\
\hline \\
\hline & \multicolumn{5}{|c|}{ Daughter(s): Fe-58 $\left(\beta^{+}, \varepsilon, 100 \%\right)$} \\
\hline$Q^{+}: 2307.9 \mathrm{keV}$ & & & & & \\
\hline \multicolumn{6}{|c|}{ Possible parent(s): Co-58m (I.T., $100 \%)$} \\
\hline \multicolumn{6}{|c|}{ Half-life (T/2): 70.85 (3) $d \equiv 6.1214(26) 10^{6} s$} \\
\hline \multicolumn{6}{|c|}{ Decay constant $(\lambda): 113.233(48) 10^{-9} \mathrm{~s}^{-1}$} \\
\hline \multicolumn{6}{|c|}{ Specific activity (Am): $1.17570(50) 10^{15} \mathrm{~Bq} \cdot \mathrm{g}^{-1}$} \\
\hline \multicolumn{6}{|c|}{ Reference: CEA/LNE-LNHB - 2013} \\
\hline \multicolumn{6}{|c|}{ Associated data files: Table - Comments - ENSDF-PenNuc } \\
\hline Intensity threshold: & & & & & \\
\hline Gamma emissions ( & es out of 4) sort & d by inc & creasing et & nergy & \\
\hline Emissions meeting & ch criteria Othe & signific & cantly inte & nse emi & issions \\
\hline & & & & Leve & \\
\hline Energy (keV) & Intensity (\%) & Type & Origin" & Start ${ }^{*}$ & End ${ }^{*}$ \\
\hline $511(-)$ & $29.88(32)$ & $y_{ \pm}$ & $\mathrm{Fe}-58$ & -1 & -1 \\
\hline $810.7602(20)$ & $99.44(2)$ & y & $\mathrm{Fe}-58$ & 1 & 0 \\
\hline
\end{tabular}

Figure 7b. LARAWEB search engine: associated data, with relevant emissions highlighted.

\section{Conclusions}

Data evaluation and dissemination is a continuous process in which the LNHB is deeply involved. We are permanently updating data and tools and developing new ones to fulfil the needs of decay data users and evaluators. Over a period of 10 years, about 100 new nuclide evaluations have been added to our database and published in six volumes of the Monographie BIPM-5, and an updated pocket-sized reference guide entitled the Mini Table of Radionuclides issued in 2015.

Many new developments have been implemented in the online tool LARAWEB, including information on $\gamma-\gamma$ coincidences and the ability to plot decay schemes. In particular, the use of intensity thresholds allows the main decay radiations to be displayed and a simplified decay scheme to be plotted, which is unique to LARAWEB.

We would like to express our sincere thanks to all members of the Decay Data Evaluation Project, past and present, without whose dedication this work would not have been possible. A full list is available from our website at: http://www.nucleide.org/ DDEP_WG/DDEPadresses.htm.

\section{References}

[1] http://www.nucleide.org/DDEP.htm

[2] M.R. Bhat, in Evaluated Nuclear Structure Data File (ENSDF), edited by S.M. Qaim, Nucl. Data Sci. Tech. (Springer-Verlag, Berlin, 1992), p. 817 ENSDF ref.

[3] http://www.bipm.org

[4] http://www.bipm.org/en/publications/ scientific-output/monographie-ri-5.html

[5] http://laboutique.edpsciences.fr/produit/ $781 / 9782759818075$ 